\title{
Newton-Kantorovich and Smale Uniform Type Convergence Theorem for a Deformed Newton Method in Banach Spaces
}

\author{
Rongfei Lin, ${ }^{1}$ Yueqing Zhao, ${ }^{1}$ Zdeněk Šmarda, ${ }^{2}$ Yasir Khan, ${ }^{3}$ and Qingbiao Wu ${ }^{3}$ \\ ${ }^{1}$ Department of Mathematics, Taizhou University, Linhai, Zhejiang 317000, China \\ ${ }^{2}$ Department of Mathematics, Faculty of Electrical Engineering and Communication, Brno University of Technology, \\ Technicka 8, 61600 Brno, Czech Republic \\ ${ }^{3}$ Department of Mathematics, Zhejiang University, Hangzhou, Zhejiang 310027, China
}

Correspondence should be addressed to Rongfei Lin; fighting.falcon2006@hotmail.com

Received 12 September 2013; Accepted 18 November 2013

Academic Editor: Miroslava Růžičková

Copyright (C) 2013 Rongfei Lin et al. This is an open access article distributed under the Creative Commons Attribution License, which permits unrestricted use, distribution, and reproduction in any medium, provided the original work is properly cited.

Newton-Kantorovich and Smale uniform type of convergence theorem of a deformed Newton method having the third-order convergence is established in a Banach space for solving nonlinear equations. The error estimate is determined to demonstrate the efficiency of our approach. The obtained results are illustrated with three examples.

\section{Introduction}

In this paper, we study the problem of approximating a unique solution $x^{*}$ of a nonlinear operator equation

$$
F(x)=0,
$$

where $F$ is a Fréchet-differentiable operator defined on an open convex $\Omega$ of a Banach space $X$ with values in a Banach space $Y$.

There are many iterative methods (see [1-3]), which have been used for finding a solution of (1). For example, the wellknown iterative method for solving (1) is Newton's method defined by

$$
x_{n+1}=x_{n}-F^{\prime}\left(x_{n}\right)^{-1} F\left(x_{n}\right), \quad(n \geq 0)\left(x_{0} \in \Omega\right) .
$$

Under the appropriate assumptions, Newton's method is the second-order convergence. Kantorovich (see [4]) presented the famous convergence result regarding a solution of (1). Many Newton-Kantorovich type of convergence theorems were given in papers [5-11]. Frontini and Sormani (see [12]) presented a new deformed Newton method with

$$
\int_{x_{n}}^{x} f^{\prime}(t) d t \simeq\left(x-x_{n}\right) f^{\prime}\left(\frac{x_{n}+x}{2}\right) .
$$

The deformed Newton method can be written as follows:

$$
x_{n+1}=x_{n}-\frac{f\left(x_{n}\right)}{f^{\prime}\left(x_{n}-f\left(x_{n}\right) / 2 f^{\prime}\left(x_{n}\right)\right)},
$$

where $f$ is a real or a complex function. In papers [13-17], the local convergence theorem has been established and the deformed method in a real or a complex space was discussed.

In the paper, we generalize the deformed Newton method [18] in a Banach space. The deformed Newton method [18] is shown as follows:

$$
\begin{gathered}
y_{n}=x_{n}-F^{\prime}\left(x_{n}\right)^{-1} F\left(x_{n}\right), \\
x_{n+1}=x_{n}-F^{\prime}\left(\frac{x_{n}+y_{n}}{2}\right)^{-1} F\left(x_{n}\right),
\end{gathered}
$$

where $F$ is defined on an open convex subset $\Omega$ of a Banach space $X$ with values in a Banach space $Y, F(x)$ has Fréchet derivatives in $\Omega$, and $F^{\prime}(x)^{-1}$ exists.

We establish Newton-Kantorovich and Smale uniform type convergence theorem (see [18]) for the deformed Newton method with the third-order in a Banach space with new sufficient conditions for the existence of a well-defined sequence which converges to a unique solution $x^{*}$ of (1). 


\section{Main Results}

Denote $g(t)=\int_{0}^{t}(t-u) L(u) d u-t+\eta, u \in(0, R), \eta>0$, and suppose $L(u), L^{\prime}(u)$ are the positive and nondecreasing continuous functions, $\lim _{t \rightarrow R^{+}} g(t)=g\left(R^{+}\right)>0, \int_{0}^{R} L(u) d u>1$, $\int_{0}^{\alpha} L(u) d u=1$ for $\alpha \in(0, R), \beta=\alpha-\int_{0}^{\alpha}(\alpha-u) L(u) d u=$ $\int_{0}^{\alpha} u L(u) d u$.

Assume that sequences $\left\{t_{n}\right\},\left\{s_{n}\right\}$ are generated by the following formulae [18]:

$$
\begin{gathered}
s_{n}=t_{n}-g^{\prime}\left(t_{n}\right)^{-1} g\left(t_{n}\right), \\
t_{n+1}=t_{n}-g^{\prime}\left(\frac{t_{n}+s_{n}}{2}\right)^{-1} g\left(t_{n}\right), \quad t_{0}=0 .
\end{gathered}
$$

Firstly, we give some lemmas.

Lemma 1. If $\eta \leq \beta$, then the function $g(t)$ has two positive real roots $r_{1}, r_{2}\left(0<r_{1} \leq \alpha \leq r_{2}<R\right)$.

Proof. Because $g(0)=\eta>0, g\left(R^{+}\right)>0$, and $g^{\prime \prime}(t)=L(t)>$ 0 , we know that $g(t)$ is the convex function for $t \in(0, R)$. Hence, $\alpha$ is a unique positive root of $g^{\prime}(t)=\int_{0}^{t} L(u) d u-1$. So, the necessary and sufficient condition that $g(t)$ has two positive roots for $t \in(0, R)$ is that the minimum of $g(t)$ satisfies the condition $g(\alpha) \leq 0$, which holds for $\eta \leq \beta$. This completes the proof of Lemma 1.

Lemma 2. Suppose the sequences $\left\{t_{n}\right\},\left\{s_{n}\right\}$ are generated by (6). Then, for $\eta \leq \beta$, the sequences $\left\{t_{n}\right\},\left\{s_{n}\right\}$ are increasing and converge to the minimum positive root of $g(t)$, and

$$
0 \leq t_{n} \leq s_{n} \leq t_{n+1}<r_{1}
$$

Proof. Denote

$$
U(x)=x-\frac{g(x)}{g^{\prime}(x)}, \quad V(x)=x-\frac{g(x)}{g^{\prime}((x+U(x)) / 2)} .
$$

On $\left[0, r_{1}\right)$, we know $g(t)>0, g^{\prime}(t)<0, g^{\prime \prime}(t)>0$, and $g^{\prime \prime}(t)$ is increasing. Denoting $y=(x+U(x)) / 2=x-$ $g(x) / 2 g^{\prime}(x)$, then

$$
\begin{gathered}
U^{\prime}(x)=\frac{g(x) g^{\prime \prime}(x)}{g^{\prime}(x)^{2}}>0, \\
{\left[g^{\prime}(y)-g^{\prime}(x)\right]=g^{\prime \prime}(\xi)(y-x)=-g^{\prime \prime}(\xi) \frac{g(x)}{2 g^{\prime}(x)},} \\
\xi \in(x, y), \\
V^{\prime}(x)=1-\left(g^{\prime}(x) g^{\prime}(y)-\frac{1}{2} g(x) g^{\prime \prime}(y)\right. \\
\left.\quad \times 1+\left(\frac{g(x) g^{\prime \prime}(x)}{g^{\prime}(x)^{2}}\right)\right)
\end{gathered}
$$

$$
\begin{aligned}
& \times\left(g^{\prime}(y)^{2}\right)^{-1} \\
= & \frac{1}{g^{\prime}(y)}\left[g^{\prime}(y)-g^{\prime}(x)\right]+\frac{g(x) g^{\prime \prime}(y)}{2 g^{\prime}(y)^{2}} \\
+ & \frac{g(x)^{2} g^{\prime \prime}(x) g^{\prime \prime}(y)}{2 g^{\prime}(x)^{2} g^{\prime}(y)^{2}} \geq-\frac{g^{\prime \prime}(\xi)}{g^{\prime}(y)} \cdot \frac{g(x)}{2 g^{\prime}(x)} \\
+ & \frac{g(x) g^{\prime \prime}(y)}{2 g^{\prime}(y)^{2}}=-\frac{g(x) g^{\prime \prime}(\xi)}{2 g^{\prime}(y)^{2} g^{\prime}(x)}\left[g^{\prime}(y)-g^{\prime}(x)\right] \\
+ & \frac{g(x) g^{\prime \prime}(y)-g(x) g^{\prime \prime}(\xi)}{2 g^{\prime}(y)^{2}}=\frac{g(x) g^{\prime \prime}(\xi)}{2 g^{\prime}(y)^{2} g^{\prime}(x)} \\
& . \frac{g(x) g^{\prime \prime}(\xi)}{2 g^{\prime}(x)}+\frac{g(x) g^{\prime \prime}(y)-g(x) g^{\prime \prime}(\xi)}{2 g^{\prime}(y)^{2}}>0 .
\end{aligned}
$$

Therefore, $U(x), V(x)$ are increasing on $\left[0, r_{1}\right]$. Thus, for $x \in\left[0, r_{1}\right), U(x)<U\left(r_{1}\right)=r_{1}, V(x)<V\left(r_{1}\right)=r_{1}$. Moreover,

$$
s_{n}=U\left(t_{n}\right), \quad t_{n+1}=V\left(t_{n}\right), \quad t_{0}=0<r_{1} ;
$$

hence we can easily prove Lemma 2 by the induction.

Suppose $X$ and $Y$ are the Banach spaces, $\Omega \subset X$ is an open convex subset, $F: \Omega \subset X \rightarrow Y$ has the secondorder Fréchet derivative, $F^{\prime}\left(x_{0}\right)^{-1}$ exists for $x_{0} \in \Omega$, and the following conditions hold:

$$
\begin{gathered}
\left\|F^{\prime}\left(x_{0}\right)^{-1} F\left(x_{0}\right)\right\| \leq \eta, \quad\left\|F^{\prime}\left(x_{0}\right)^{-1} F^{\prime \prime}\left(x_{0}\right)\right\| \leq L(0), \\
\left\|F^{\prime}\left(x_{0}\right)^{-1}\left(F^{\prime \prime}(y)-F^{\prime \prime}(x)\right)\right\| \\
\quad \leq \int_{\rho(x)}^{\rho(\overline{x, y})} L^{\prime}(u) d u, \quad x, y \in \Omega, \rho(\overline{x, y})<\alpha,
\end{gathered}
$$

where $\rho(x)=\left\|x-x_{0}\right\|$ and $\rho(\overline{x, y})=\|y-x\|+\left\|x-x_{0}\right\|$.

Lemma 3. Suppose $F$ satisfies (11) and $\left\|x-x_{0}\right\|<\alpha$. Then $F^{\prime}(x)^{-1}$ exists, and

$$
\begin{aligned}
& \left\|F^{\prime}\left(x_{0}\right)^{-1} F^{\prime \prime}(x)\right\| \leq g^{\prime \prime}\left(\left\|x-x_{0}\right\|\right), \\
& \left\|F^{\prime}(x)^{-1} F^{\prime}\left(x_{0}\right)\right\| \leq-\frac{1}{g^{\prime}\left(\left\|x-x_{0}\right\|\right)} .
\end{aligned}
$$

Proof. Firstly, by the conditions (11), we know that

$$
\begin{aligned}
\left\|F^{\prime}\left(x_{0}\right)^{-1} F^{\prime \prime}(x)\right\| \leq & \left\|F^{\prime}\left(x_{0}\right)^{-1} F^{\prime \prime}\left(x_{0}\right)\right\| \\
& +\left\|F^{\prime}\left(x_{0}\right)^{-1} F^{\prime \prime}(x)-F^{\prime}\left(x_{0}\right)^{-1} F^{\prime \prime}\left(x_{0}\right)\right\| \\
\leq & L(0)+\int_{0}^{\left\|x-x_{0}\right\|} L^{\prime}(u) d u \\
& =L\left(\left\|x-x_{0}\right\|\right)=g^{\prime \prime}\left(\left\|x-x_{0}\right\|\right) .
\end{aligned}
$$


Secondly, we know $g^{\prime}(t)<0$ for $t<\alpha$. Hence

$$
\begin{aligned}
\left\|F^{\prime}\left(x_{0}\right)^{-1} F^{\prime}(x)-I\right\| & =\left\|F^{\prime}\left(x_{0}\right)^{-1}\left[F^{\prime}(x)-F^{\prime}\left(x_{0}\right)\right]\right\| \\
& =\| F^{\prime}\left(x_{0}\right)^{-1} \int_{0}^{1} F^{\prime \prime}\left(x_{0}+t\left(x-x_{0}\right)\right) \\
& \times\left(x-x_{0}\right) d t \| \\
& \leq \int_{0}^{1} g^{\prime \prime}\left(t\left\|x-x_{0}\right\|\right)\left\|x-x_{0}\right\| d t \\
& =g^{\prime}\left(\left\|x-x_{0}\right\|\right)-g^{\prime}(0) \\
& =g^{\prime}\left(\left\|x-x_{0}\right\|\right)+1<1 .
\end{aligned}
$$

By Banach Theorem, we know $F^{\prime}(x)^{-1}$ exists, and

$$
\begin{aligned}
\left\|F^{\prime}(x)^{-1} F^{\prime}\left(x_{0}\right)\right\| & \leq \frac{1}{1-\left\|F^{\prime}\left(x_{0}\right)^{-1} F^{\prime}(x)-I\right\|} \\
& =-\frac{1}{g^{\prime}\left(\left\|x-x_{0}\right\|\right)} .
\end{aligned}
$$

This completes the proof of Lemma 3.

Lemma 4. Suppose $X$ and $Y$ are Banach spaces, $\Omega$ is an open convex of the Banach space $X, F: \Omega \subset X \rightarrow Y$ has the second-order Fréchet derivative, and the sequences $\left\{x_{n}\right\}$, $\left\{y_{n}\right\}$ are generated by (5). Then, for any natural number $n$, the following formula holds:

$$
\begin{aligned}
F\left(x_{n+1}\right) & \\
= & \int_{0}^{1} F^{\prime \prime}\left(\frac{x_{n}+y_{n}}{2}+t\left(x_{n+1}-\frac{x_{n}+y_{n}}{2}\right)\right)(1-t) d t \\
& \times\left(x_{n+1}-y_{n}\right)^{2} \\
& +\frac{1}{2} \int_{0}^{1} F^{\prime \prime}\left(\frac{x_{n}+y_{n}}{2}+t\left(x_{n+1}-\frac{x_{n}+y_{n}}{2}\right)\right)(1-t) d t \\
& \times\left(x_{n+1}-y_{n}\right)\left(y_{n}-x_{n}\right) \\
& +\frac{1}{2} \int_{0}^{1} F^{\prime \prime}\left(\frac{x_{n}+y_{n}}{2}+t\left(x_{n+1}-\frac{x_{n}+y_{n}}{2}\right)\right)(1-t) d t \\
& \times\left(y_{n}-x_{n}\right)\left(x_{n+1}-y_{n}\right) \\
& +\frac{1}{4} \int_{0}^{1} F^{\prime \prime}\left(\frac{x_{n}+y_{n}}{2}+t\left(x_{n+1}-\frac{x_{n}+y_{n}}{2}\right)\right)(1-t) d t \\
& \times\left(y_{n}-x_{n}\right)^{2} \\
& -\frac{1}{4} \int_{0}^{1} F^{\prime \prime}\left(\frac{x_{n}+y_{n}}{2}-t\left(\frac{y_{n}-x_{n}}{2}\right)\right)(1-t) d t \\
& \times\left(y_{n}-x_{n}\right)^{2} .
\end{aligned}
$$

Proof. By (5), we have

$$
\begin{aligned}
F\left(x_{n+1}\right)= & F\left(x_{n+1}\right)-F\left(\frac{x_{n}+y_{n}}{2}\right) \\
& -F^{\prime}\left(\frac{x_{n}+y_{n}}{2}\right)\left(x_{n+1}-\frac{x_{n}+y_{n}}{2}\right) \\
& +F\left(\frac{x_{n}+y_{n}}{2}\right)+F^{\prime}\left(\frac{x_{n}+y_{n}}{2}\right) \\
& \times\left(x_{n+1}-\frac{x_{n}+y_{n}}{2}\right) \\
= & \int_{0}^{1} F^{\prime \prime}\left(\frac{x_{n}+y_{n}}{2}+t\left(x_{n+1}-\frac{x_{n}+y_{n}}{2}\right)\right)(1-t) d t \\
& \times\left(x_{n+1}-\frac{x_{n}+y_{n}}{2}\right)^{2}+F\left(\frac{x_{n}+y_{n}}{2}\right) \\
& +F^{\prime}\left(\frac{x_{n}+y_{n}}{2}\right)\left(x_{n+1}-\frac{x_{n}+y_{n}}{2}\right), \\
F & \left(\frac{x_{n}+y_{n}}{2}\right)+F^{\prime}\left(\frac{x_{n}+y_{n}}{2}\right)\left(x_{n+1}-\frac{x_{n}+y_{n}}{2}\right) \\
= & F\left(\frac{x_{n}+y_{n}}{2}\right)+F^{\prime}\left(\frac{x_{n}+y_{n}}{2}\right) \\
& \times\left(x_{n+1}-x_{n}-\frac{y_{n}-x_{n}}{2}\right) \\
= & F\left(\frac{x_{n}+y_{n}}{2}\right)-F\left(x_{n}\right)-F^{\prime}\left(\frac{x_{n}+y_{n}}{2}\right) \frac{y_{n}-x_{n}}{2} \\
= & -\frac{1}{4} \int_{0}^{1} F^{\prime \prime}\left(\frac{x_{n}+y_{n}}{2}-t\left(\frac{y_{n}-x_{n}}{2}\right)\right)(1-t) d t \\
& \times\left(y_{n}-x_{n}\right)^{2} .
\end{aligned}
$$

Hence

$$
\begin{aligned}
F\left(x_{n+1}\right)= & \int_{0}^{1} F^{\prime \prime}\left(\frac{x_{n}+y_{n}}{2}+t\left(x_{n+1}-\frac{x_{n}+y_{n}}{2}\right)\right) \\
& \times(1-t) d t\left(x_{n+1}-y_{n}\right)^{2} \\
+ & \frac{1}{2} \int_{0}^{1} F^{\prime \prime}\left(\frac{x_{n}+y_{n}}{2}+t\left(x_{n+1}-\frac{x_{n}+y_{n}}{2}\right)\right) \\
& \times(1-t) d t\left(x_{n+1}-y_{n}\right)\left(y_{n}-x_{n}\right) \\
+ & \frac{1}{2} \int_{0}^{1} F^{\prime \prime}\left(\frac{x_{n}+y_{n}}{2}+t\left(x_{n+1}-\frac{x_{n}+y_{n}}{2}\right)\right) \\
& \times(1-t) d t\left(y_{n}-x_{n}\right)\left(x_{n+1}-y_{n}\right) \\
+ & \frac{1}{4} \int_{0}^{1} F^{\prime \prime}\left(\frac{x_{n}+y_{n}}{2}+t\left(x_{n+1}-\frac{x_{n}+y_{n}}{2}\right)\right) \\
& \times(1-t) d t\left(y_{n}-x_{n}\right)^{2}
\end{aligned}
$$




$$
\begin{aligned}
& -\frac{1}{4} \int_{0}^{1} F^{\prime \prime}\left(\frac{x_{n}+y_{n}}{2}-t\left(\frac{y_{n}-x_{n}}{2}\right)\right)(1-t) d t \\
& \times\left(y_{n}-x_{n}\right)^{2} .
\end{aligned}
$$

This completes the proof of Lemma 4.

Theorem 5. Suppose $X$ and $Y$ are Banach spaces, $\Omega \subset X$ is an open convex subset, $F: \Omega \subset X \rightarrow Y$ satisfies condition (11), $\eta \leq \beta$, and

$$
\overline{S\left(x_{0}, r_{1}\right)}=\left\{x \mid\left\|x-x_{0}\right\| \leq r_{1}, x \in X\right\} \subset \Omega \text {. }
$$

Then the sequence $\left\{x_{n}\right\}_{n \geq 0}$ generated by (5) is well defined, $x_{n} \in$ $\overline{S\left(x_{0}, r_{1}\right)}$, and converges to the unique solution $x^{*}$ in $S\left(x_{0}, \alpha\right)$ and

$$
\left\|x_{n}-x^{*}\right\| \leq r_{1}-t_{n}
$$

Proof. By induction, we can prove that the following formulae hold:

$$
\begin{gathered}
\left\|x_{n}-x_{0}\right\| \leq t_{n} ; \\
\left\|F^{\prime}\left(x_{n}\right)^{-1} F^{\prime}\left(x_{0}\right)\right\| \leq-g^{\prime}\left(t_{n}\right)^{-1} ; \\
\left\|y_{n}-x_{n}\right\| \leq s_{n}-t_{n} ; \\
\left\|y_{n}-x_{0}\right\| \leq s_{n} ; \\
\left\|F^{\prime}\left(\frac{x_{n}+y_{n}}{2}\right)^{-1} F^{\prime}\left(x_{0}\right)\right\| \leq-g^{\prime}\left(\frac{t_{n}+s_{n}}{2}\right)^{-1} ; \\
\left\|x_{n+1}-y_{n}\right\| \leq t_{n+1}-s_{n} ; \\
\left\|x_{n+1}-x_{n}\right\| \leq t_{n+1}-t_{n} .
\end{gathered}
$$

In fact, by Lemma 2 we know $t_{n}<r_{1}$ for any natural number $n$. It is easy to prove that for $n=0$ the above formulae hold. Suppose the above formulae also hold for $n>0$. Then

$$
\begin{aligned}
\left\|x_{n+1}-x_{0}\right\| \leq & \left\|x_{n+1}-x_{n}\right\| \\
& +\left\|x_{n}-x_{0}\right\| \leq t_{n+1}-t_{n}+t_{n}=t_{n+1} .
\end{aligned}
$$

By Lemma 3, we get

$$
\begin{aligned}
\left\|F^{\prime}\left(x_{n+1}\right)^{-1} F^{\prime}\left(x_{0}\right)\right\| & \leq-g^{\prime}\left(\left\|x_{n+1}-x_{0}\right\|\right)^{-1} \\
& \leq-g^{\prime}\left(t_{n+1}\right)^{-1} .
\end{aligned}
$$

By Lemmas 3 and 4 and the fact that $-g^{\prime}(t)^{-1}, g^{\prime \prime}(t)$ are positive and increasing on $[0, \alpha)$, we have

$$
\begin{aligned}
& \| F^{\prime}\left(x_{0}\right)^{-1}\left[F^{\prime \prime}\left(\frac{x_{n}+y_{n}}{2}+t\left(x_{n+1}-\frac{x_{n}+y_{n}}{2}\right)\right)\right. \\
&\left.\quad-F^{\prime \prime}\left(\frac{x_{n}+y_{n}}{2}-t\left(\frac{y_{n}-x_{n}}{2}\right)\right)\right] \| \\
& \leq \int_{0}^{t\left\|x_{n+1}-x_{n}\right\|} L^{\prime}\left(u+\left\|\frac{x_{n}+y_{n}}{2}-t\left(\frac{y_{n}-x_{n}}{2}\right)-x_{0}\right\|\right) d u \\
& \leq \int_{0}^{t\left(t_{n+1}-t_{n}\right)} L^{\prime}\left(u+\frac{t_{n}+s_{n}}{2}-t \frac{s_{n}-t_{n}}{2}\right) d u \\
&= L\left(\frac{t_{n}+s_{n}}{2}+t\left(t_{n+1}-\frac{t_{n}+s_{n}}{2}\right)\right) \\
&-L\left(\frac{t_{n}+s_{n}}{2}-t\left(\frac{s_{n}-t_{n}}{2}\right)\right) \\
&= g^{\prime \prime}\left(\frac{t_{n}+s_{n}}{2}+t\left(t_{n+1}-\frac{t_{n}+s_{n}}{2}\right)\right) \\
&-g^{\prime \prime}\left(\frac{t_{n}+s_{n}}{2}-t\left(\frac{s_{n}-t_{n}}{2}\right)\right),
\end{aligned}
$$

$$
\left\|F^{\prime}\left(x_{0}\right)^{-1} F\left(x_{n+1}\right)\right\|
$$

$$
\begin{aligned}
\leq & \int_{0}^{1} g^{\prime \prime}\left(\frac{t_{n}+s_{n}}{2}+t\left(t_{n+1}-\frac{t_{n}+s_{n}}{2}\right)\right) \\
& \times(1-t) d t\left(t_{n+1}-s_{n}\right)^{2} \\
& +\frac{1}{2} \int_{0}^{1} g^{\prime \prime}\left(\frac{t_{n}+s_{n}}{2}+t\left(t_{n+1}-\frac{t_{n}+s_{n}}{2}\right)\right)(1-t) d t \\
& \times\left(t_{n+1}-s_{n}\right)\left(s_{n}-t_{n}\right) \\
& +\frac{1}{2} \int_{0}^{1} g^{\prime \prime}\left(\frac{t_{n}+s_{n}}{2}+t\left(t_{n+1}-\frac{t_{n}+s_{n}}{2}\right)\right)(1-t) d t \\
& \times\left(s_{n}-t_{n}\right)\left(t_{n+1}-s_{n}\right) \\
& +\frac{1}{4} \int_{0}^{1} g^{\prime \prime}\left(\frac{t_{n}+s_{n}}{2}+t\left(t_{n+1}-\frac{t_{n}+s_{n}}{2}\right)\right)(1-t) d t \\
& \times\left(s_{n}-t_{n}\right)^{2} \\
& -\frac{1}{4} \int_{0}^{1} g^{\prime \prime}\left(\frac{t_{n}+s_{n}}{2}-t\left(\frac{s_{n}-t_{n}}{2}\right)\right)(1-t) d t \\
& \times\left(s_{n}-t_{n}\right)^{2}=g\left(t_{n+1}\right) .
\end{aligned}
$$

Hence we get

$$
\begin{aligned}
\left\|y_{n+1}-x_{n+1}\right\|= & \left\|-F^{\prime}\left(x_{n+1}\right)^{-1} F\left(x_{n+1}\right)\right\| \\
\leq & \left\|-F^{\prime}\left(x_{n+1}\right)^{-1} F^{\prime}\left(x_{0}\right)\right\| \\
& \times\left\|F^{\prime}\left(x_{0}\right)^{-1} F\left(x_{n+1}\right)\right\|
\end{aligned}
$$




$$
\begin{aligned}
\leq & -g^{\prime}\left(t_{n+1}\right)^{-1} g\left(t_{n+1}\right) \\
= & s_{n+1}-t_{n+1}, \\
\left\|y_{n+1}-x_{0}\right\| \leq & \left\|y_{n+1}-x_{n+1}\right\| \\
& +\left\|x_{n+1}-x_{0}\right\| \leq s_{n+1} .
\end{aligned}
$$

By Lemma 3, we get

$$
\left\|F^{\prime}\left(\frac{x_{n+1}+y_{n+1}}{2}\right)^{-1} F^{\prime}\left(x_{0}\right)\right\| \leq-g^{\prime}\left(\frac{t_{n+1}+s_{n+1}}{2}\right)^{-1} .
$$

Moreover, we have

$$
\begin{aligned}
& \left\|x_{n+2}-y_{n+1}\right\| \\
& =\| F^{\prime}\left(x_{n+1}\right)^{-1} F\left(x_{n+1}\right) \\
& -F^{\prime}\left(\frac{x_{n+1}+y_{n+1}}{2}\right)^{-1} F\left(x_{n+1}\right) \| \\
& =\| F^{\prime}\left(\frac{x_{n+1}+y_{n+1}}{2}\right)^{-1}\left[F^{\prime}\left(\frac{x_{n+1}+y_{n+1}}{2}\right)\right. \\
& \left.-F^{\prime}\left(x_{n+1}\right)\right] \\
& \times F^{\prime}\left(x_{n+1}\right)^{-1} F\left(x_{n+1}\right) \| \\
& =\| F^{\prime}\left(\frac{x_{n+1}+y_{n+1}}{2}\right)^{-1} F^{\prime}\left(x_{0}\right) F^{\prime}\left(x_{0}\right)^{-1} \\
& \times \int_{0}^{1} F^{\prime \prime}\left(x_{n+1}+\frac{t}{2}\left(y_{n+1}-x_{n+1}\right)\right) d t \\
& \times \frac{y_{n+1}-x_{n+1}}{2} F^{\prime}\left(x_{n+1}\right)^{-1} \\
& \times F^{\prime}\left(x_{0}\right) F^{\prime}\left(x_{0}\right)^{-1} F\left(x_{n+1}\right) \| \\
& \leq g^{\prime}\left(\frac{t_{n+1}+s_{n+1}}{2}\right)^{-1} \\
& \times \int_{0}^{1} g^{\prime \prime}\left(t_{n+1}+\frac{t}{2}\left(s_{n+1}-t_{n+1}\right)\right) d t \\
& \times \frac{\left(s_{n+1}-t_{n+1}\right)}{2} g^{\prime}\left(t_{n+1}\right)^{-1} g\left(t_{n+1}\right) \\
& \leq g^{\prime}\left(\frac{t_{n+1}+s_{n+1}}{2}\right)^{-1} \\
& \times\left[g^{\prime}\left(\frac{t_{n+1}+s_{n+1}}{2}\right)-g^{\prime}\left(t_{n+1}\right)\right] \\
& \times g^{\prime}\left(t_{n+1}\right)^{-1} g\left(t_{n+1}\right)
\end{aligned}
$$

$$
\begin{aligned}
= & g^{\prime}\left(t_{n+1}\right)^{-1} g\left(t_{n+1}\right) \\
& -g^{\prime}\left(\frac{t_{n+1}+s_{n+1}}{2}\right)^{-1} \\
& \times g\left(t_{n+1}\right)=t_{n+2}-s_{n+1},
\end{aligned}
$$

$$
\begin{aligned}
\left\|x_{n+2}-x_{n+1}\right\| \leq & \left\|x_{n+2}-y_{n+1}\right\| \\
& +\left\|y_{n+1}-x_{n+1}\right\| \leq t_{n+2}-t_{n+1} .
\end{aligned}
$$

Hence, the sequence $\left\{x_{n}\right\}_{n \geq 0}$ generated by (5) is well defined, $x_{n} \in \overline{S\left(x_{0}, r_{1}\right)}$, and $\left\{x_{n}\right\}$ converges to the solution $x^{*} \in \overline{S\left(x_{0}, r_{1}\right)}$ of $(1)$.

Now we prove the uniqueness. Suppose $y^{*}$ is also a solution of (1) on $S\left(x_{0}, \alpha\right)$. We know that $g^{\prime}(t)<0$ for $t \in[0, \alpha)$. Then

$$
\begin{aligned}
& \left\|F^{\prime}\left(x_{0}\right)^{-1} \int_{0}^{1} F^{\prime}\left(x^{*}+t\left(y^{*}-x^{*}\right)\right) d t-I\right\| \\
& \leq\left\|F^{\prime}\left(x_{0}\right)^{-1} \int_{0}^{1}\left\{F^{\prime}\left[x^{*}+t\left(y^{*}-x^{*}\right)\right]-F^{\prime}\left(x_{0}\right)\right\} d t\right\| \\
& \leq \| F^{\prime}\left(x_{0}\right)^{-1} \int_{0}^{1} \int_{0}^{1} F^{\prime \prime}\left(x_{0}+s\left(x^{*}-x_{0}+t\left(y^{*}-x^{*}\right)\right)\right) d s d t \\
& \quad \times\left(x^{*}-x_{0}+t\left(y^{*}-x^{*}\right)\right) \| \\
& \leq \int_{0}^{1} \int_{0}^{1} g^{\prime \prime}\left(s\left\|x^{*}-x_{0}+t\left(y^{*}-x^{*}\right)\right\|\right) d s d t \\
& \quad \times\left\|x^{*}-x_{0}+t\left(y^{*}-x^{*}\right)\right\| \\
& =\int_{0}^{1} g^{\prime}\left(\left\|x^{*}-x_{0}+t\left(y^{*}-x^{*}\right)\right\|\right) d t-g^{\prime}(0) \\
& =\int_{0}^{1} g^{\prime}\left(\left\|(1-t)\left(x^{*}-x_{0}\right)+t\left(y^{*}-x_{0}\right)\right\|\right) d t+1<1 .
\end{aligned}
$$

By Banach Theorem, we know the inverse of $\int_{0}^{1} F^{\prime}\left[x^{*}+\right.$ $\left.t\left(y^{*}-x^{*}\right)\right] d t$ exists and

$$
\begin{aligned}
0 & =F\left(y^{*}\right)-F\left(x^{*}\right) \\
& =\int_{0}^{1} F^{\prime}\left[x^{*}+t\left(y^{*}-x^{*}\right)\right] d t\left(y^{*}-x^{*}\right) ;
\end{aligned}
$$

hence we get $y^{*}=x^{*}$. This completes the proof of the uniqueness of the solution of (1).

For $m>n$, we know that

$$
\begin{aligned}
\left\|x_{m}-x_{n}\right\| \leq & \left\|x_{m}-x_{m-1}\right\| \\
& +\left\|x_{m-1}-x_{m-2}\right\|+\cdots+\left\|x_{n+1}-x_{n}\right\| \leq t_{m}-t_{n} .
\end{aligned}
$$

When $m \rightarrow \infty$, we get

$$
\left\|x_{n}-x^{*}\right\| \leq r_{1}-t_{n} .
$$

This completes the proof of Theorem 5 . 
Suppose that $L(u)=\gamma+K u, u \in(0,+\infty), \gamma, K>0$. Then $\int_{\rho(x)}^{\rho(\overline{x, y)}} L^{\prime}(u) d u=K\|x-y\|, g(t)=(1 / 6) K t^{3}+(1 / 2) \gamma t^{2}-t+$ $\eta \alpha=2 /\left(\gamma+\sqrt{\gamma^{2}+2 K}\right)$, and $\beta=\alpha-(1 / 6) K \alpha^{3}-(1 / 2) \gamma \alpha^{2}=$ $2\left(\gamma+2 \sqrt{\gamma^{2}+2 K}\right) / 3\left(\gamma+\sqrt{\gamma^{2}+2 K}\right)^{2}$.

Corollary 6. Suppose $X$ and $Y$ are the Banach spaces, $\Omega$ is an open convex subset of the Banach space $X, F: \Omega \subset X \rightarrow Y$ has the second-order Fréchet derivative, $F^{\prime}\left(x_{0}\right)^{-1}$ exists for $x_{0} \in \Omega$, and the following conditions hold:

$$
\begin{gathered}
\left\|F^{\prime}\left(x_{0}\right)^{-1} F\left(x_{0}\right)\right\| \leq \eta, \quad\left\|F^{\prime}\left(x_{0}\right)^{-1} F^{\prime \prime}\left(x_{0}\right)\right\| \leq \gamma, \\
\left\|F^{\prime}\left(x_{0}\right)^{-1}\left(F^{\prime \prime}(x)-F^{\prime \prime}(y)\right)\right\| \leq K\|x-y\| \quad x, y \in \Omega, \\
\eta \leq \frac{2\left(\gamma+2 \sqrt{\gamma^{2}+2 K}\right)}{3\left(\gamma+\sqrt{\gamma^{2}+2 K}\right)^{2}}, \quad \overline{S\left(x_{0}, r_{1}\right)} \subset \Omega .
\end{gathered}
$$

Then the sequence $\left\{x_{n}\right\}_{n \geq 0}$ generated by (5) is well defined, $x_{n} \in \overline{S\left(x_{0}, r_{1}\right)}$, and $\left\{x_{n}\right\}$ converges to the unique solution $x^{*}$ on $S\left(x_{0}, \alpha\right)$ of $(1)$, where $r_{1} \leq r_{2}$ are two positive roots of $g(t)=$ $(1 / 6) K t^{3}+(1 / 2) \gamma t^{2}-t+\eta$.

Suppose $L(u)=2 \gamma(1-\gamma u)^{-3}, u \in(0,1 / \gamma), \quad g(t)=$ $\eta-t+\gamma t^{2} /(1-\gamma t), \alpha=(1-\sqrt{2} / 2)(1 / \gamma)$ and $\beta=(3-2 \sqrt{2}) / \gamma$ and for $\left\|x-x_{0}\right\|<\alpha,\left\|F^{\prime}\left(x_{0}\right)^{-1} F^{\prime \prime \prime}(x)\right\| \leq 6 \gamma^{2} /\left(1-\gamma\left\|x-x_{0}\right\|\right)^{4}$. Hence, for $\left\|x-x_{0}\right\|+\|y-x\|<\alpha$, we get

$$
\begin{aligned}
& \left\|F^{\prime}\left(x_{0}\right)^{-1}\left[F^{\prime \prime}(y)-F^{\prime \prime}(x)\right]\right\| \\
& =\left\|\int_{0}^{1} F^{\prime}\left(x_{0}\right)^{-1} F^{\prime \prime \prime}(x+t(y-x)) d t(y-x)\right\| \\
& \leq \int_{0}^{1} \frac{6 \gamma^{2}}{\left[1-\gamma\left\|x-x_{0}+t(y-x)\right\|\right]^{4}} d t\|y-x\| \\
& \leq \int_{0}^{1} \frac{6 \gamma^{2}}{\left[1-\gamma\left(\left\|x-x_{0}\right\|+t\|y-x\|\right)\right]^{4}} d t\|y-x\| \\
& =\int_{\left\|x-x_{0}\right\|}^{\left\|x-x_{0}\right\|+\|y-x\|} \frac{6 \gamma^{2}}{(1-\gamma u)^{4}} u \\
& =\int_{\left\|x-x_{0}\right\|}^{\left\|x-x_{0}\right\|+\|y-x\|} L^{\prime}(u) d u .
\end{aligned}
$$

Corollary 7 (see [10]). Suppose $X$ and $Y$ are Banach spaces, $\Omega$ is an open convex subset of the Banach space $X, F: \Omega \subset$
$X \rightarrow Y$ has the third-order Fréchet derivative, $F^{\prime}\left(x_{0}\right)^{-1}$ exists for $x_{0} \in \Omega$, and the following conditions hold:

$$
\begin{aligned}
& \left\|F^{\prime}\left(x_{0}\right)^{-1} F\left(x_{0}\right)\right\| \leq \eta, \quad\left\|F^{\prime}\left(x_{0}\right)^{-1} F^{\prime \prime}\left(x_{0}\right)\right\| \leq 2 \gamma, \\
& \left\|F^{\prime}\left(x_{0}\right)^{-1} F^{\prime \prime \prime}(x)\right\| \leq \frac{6 \gamma^{2}}{\left(1-\gamma\left\|x-x_{0}\right\|\right)^{4}} \\
& =g^{\prime \prime \prime}\left(\left\|x-x_{0}\right\|\right), \quad x \in \Omega, \\
& \left\|x-x_{0}\right\| \leq\left(1-\frac{1}{\sqrt{2}}\right) \frac{1}{\gamma}, \quad \eta \gamma \leq 3-2 \sqrt{2}, \\
& \overline{S\left(x_{0}, r_{1}\right)} \subset \Omega \text {. }
\end{aligned}
$$

Then the sequence $\left\{x_{n}\right\}_{n \geq 0}$ generated by (5) is well defined, $x_{n} \in \overline{S\left(x_{0}, r_{1}\right)}$, and $\left\{x_{n}\right\}$ converges to the unique solution $x^{*}$ of (1) on $S\left(x_{0},(1-1 / \sqrt{2})(1 / \gamma)\right)$, where

$$
\begin{aligned}
& r_{1}=\frac{1+\eta \gamma-\sqrt{(1+\eta \gamma)^{2}-8 \eta \gamma}}{4 \gamma}, \\
& r_{2}=\frac{1+\eta \gamma+\sqrt{(1+\eta \gamma)^{2}-8 \eta \gamma}}{4 \gamma}
\end{aligned}
$$

are two positive roots of the equation $g(t)=\eta-t+\gamma t^{2} /(1-\gamma t)$.

\section{Numerical Examples}

In this section, we apply the convergence theorem and show three numerical examples.

Example 1. Consider the equation

$$
F(x)=\frac{1}{6} x^{3}+\frac{1}{6} x^{2}-\frac{5}{6} x+\frac{1}{3}=0 .
$$

We choose the initial point $x_{0}=0, \Omega=[-1,1]$; then

$$
\begin{gathered}
\eta=\left|F^{\prime}(0)^{-1} F(0)\right|=\frac{2}{5}, \quad \gamma=\left|F^{\prime}(0)^{-1} F^{\prime \prime}(0)\right|=\frac{2}{5}, \\
K=\frac{6}{5}, \\
\frac{2\left(\gamma+2 \sqrt{\gamma^{2}+2 K}\right)}{3\left(\gamma+\sqrt{\gamma^{2}+2 K}\right)^{2}}=\frac{3}{5}>\eta .
\end{gathered}
$$

Hence, by Corollary 6 , the sequence $\left\{x_{n}\right\}_{n \geq 0}$ generated by (5) is well defined, and $\left\{x_{n}\right\}$ converges to the solution $x^{*}$ of (36).

Now, we will analyze errors $\left\|x_{n}-x^{*}\right\|$ by Corollary 6 (see Table 1). In this case, we take $x_{0}=0$; then $r_{1}=$ $0.462598422 \cdots$.

Example 2. Consider the system of equation [18] $F(u, v)=0$, where

$$
F(u, v)=(u v-1, u v+u-2 v)^{T} .
$$


TABLE 1: Error results for Corollary $6\left(\left\|x_{n}-x^{*}\right\| \leq r_{1}-t_{n}\right)$.

\begin{tabular}{lccc}
\hline Step & $r_{1}-t_{n}$ & Step & $r_{1}-t_{n}$ \\
\hline$k=1$ & $1.616985 \times 10^{-2}$ & $k=2$ & $2.236349 \times 10^{-6}$ \\
$k=3$ & $6.225929 \times 10^{-18}$ & $k=4$ & $1.343387 \times 10^{-52}$ \\
$k=5$ & $1.349560 \times 10^{-156}$ & $k=6$ & $1.368249 \times 10^{-468}$ \\
\hline
\end{tabular}

Then, we have

$$
\begin{gathered}
F^{\prime}(u, v)=\left(\begin{array}{cc}
v & u \\
v+1 & u-2
\end{array}\right), \\
F^{\prime}(u, v)^{-1}=-\frac{1}{u+2 v}\left(\begin{array}{cc}
u-2 & -u \\
-v-1 & v
\end{array}\right), \\
F^{\prime \prime}(u, v)=\left(\begin{array}{cc}
0 & 1 \\
1 & 0 \\
\hline 0 & 1 \\
1 & 0
\end{array}\right) .
\end{gathered}
$$

We choose $x_{0}=\left(u_{0}, v_{0}\right)=(1.75,1.75)$ and $\Omega=\{x \mid$ $\left.\left\|x-x_{0}\right\| \leq 1.75\right\}$. We take the max-norm in $R^{2}$ and the norm $\|A\|=\max \left\{\left|a_{11}\right|+\left|a_{12}\right|,\left|a_{21}\right|+\left|a_{22}\right|\right\}$ for $A=\left(\begin{array}{ll}a_{11} & a_{12} \\ a_{21} & a_{22}\end{array}\right)$. Define the norm of a bilinear operator $B$ on $R^{2}$ by

$$
\|B\|=\sup _{\|u\|=1} \max _{i} \sum_{j=1}^{2}\left|\sum_{k=1}^{2} b_{i}^{j k} u_{k}\right|,
$$

where $u=\left(u_{1}, u_{2}\right)^{T}$ and

$$
B=\left(\begin{array}{ll}
b_{1}^{11} & b_{1}^{12} \\
b_{1}^{21} & b_{1}^{22} \\
\hline b_{2}^{11} & b_{2}^{12} \\
b_{2}^{21} & b_{2}^{22}
\end{array}\right)
$$

Then we get the following results:

$$
\begin{gathered}
\eta=\left\|F^{\prime}\left(x_{0}\right)^{-1} F\left(x_{0}\right)\right\|=\frac{9}{14}, \\
\gamma=\left\|F^{\prime}\left(x_{0}\right)^{-1} F^{\prime \prime}\left(x_{0}\right)\right\|=\frac{16}{21}, \\
K=0, \quad \frac{2\left(\gamma+2 \sqrt{\gamma^{2}+2 K}\right)}{3\left(\gamma+\sqrt{\gamma^{2}+2 K}\right)^{2}}>\eta .
\end{gathered}
$$
fied.

This means that the hypotheses of Corollary 6 are satis-

Now, we will analyze errors $\left\|x_{n}-x^{*}\right\|$ by Corollary 6 (see Table 2). In this case, we take $x_{0}=\left(u_{0}, v_{0}\right)=(1.75,1.75)$; then $r_{1}=1.125$.

Example 3. Consider the following integral equations:

$$
x(s)=1+\frac{1}{4} x(s) \int_{0}^{1} \frac{s}{s+t} x(t) d t
$$

TABLE 2: Error results for Corollary $6\left(\left\|x_{n}-x^{*}\right\| \leq r_{1}-t_{n}\right)$.

\begin{tabular}{lccc}
\hline Step & $r_{1}-t_{n}$ & Step & $r_{1}-t_{n}$ \\
\hline$k=1$ & $2.736486 \times 10^{-1}$ & $k=2$ & $3.044252 \times 10^{-2}$ \\
$k=3$ & $1.588069 \times 10^{-4}$ & $k=4$ & $2.844419 \times 10^{-11}$ \\
$k=5$ & $1.636509 \times 10^{-30}$ & $k=6$ & $3.116680 \times 10^{-92}$ \\
\hline
\end{tabular}

TABLE 3: Error results for Corollary $7\left(\left\|x_{n}-x^{*}\right\| \leq r_{1}-t_{n}\right)$.

\begin{tabular}{lccc}
\hline Step & $r_{1}-t_{n}$ & Step & $r_{1}-t_{n}$ \\
\hline$k=1$ & $2.764303 \times 10^{-3}$ & $k=2$ & $4.099223 \times 10^{-9}$ \\
$k=3$ & $1.344301 \times 10^{-26}$ & $k=4$ & $4.741124 \times 10^{-79}$ \\
$k=5$ & $2.079868 \times 10^{-236}$ & $k=6$ & $<1.0 \times 10^{-500}$ \\
\hline
\end{tabular}

and the space $X=C[0,1]$ with the norm

$$
\|x\|=\max _{0 \leq s \leq 1}|x(s)| \text {. }
$$

This equation arises in the theory of radiative transfer and neutron transport and in the kinetic theory of gases. Define the operator $F$ on $X$ by

$$
F(x)=\frac{1}{4} x(s) \int_{0}^{1} \frac{s}{s+t} x(t) d t-x(s)+1 .
$$

Then, for $x_{0}=1$, we obtain

$$
\begin{gathered}
\eta=\left\|F^{\prime}\left(x_{0}\right)^{-1} F\left(x_{0}\right)\right\|=0.2652, \\
2 \gamma=\left\|F^{\prime}\left(x_{0}\right)^{-1} F^{\prime \prime}\left(x_{0}\right)\right\|=1.5304 \times 2 \\
\cdot \frac{1}{4} \max _{0 \leq s \leq 1}\left|\int_{0}^{1} \frac{s}{s+t} d t\right|=1.5304 \times \frac{\ln 2}{2}=0.5303, \\
\eta \gamma=0.07032<3-2 \sqrt{2}, \\
\left\|F^{\prime}\left(x_{0}\right)^{-1} F^{\prime \prime \prime}(x)\right\|=0<\frac{6 \gamma^{2}}{\left(1-\gamma\left\|x-x_{0}\right\|\right)^{4}} .
\end{gathered}
$$

This means that the hypotheses of Corollary 7 are satisfied.

Now, we will analyze errors $\left\|x_{n}-x^{*}\right\|$ by Corollary 7 (see Table 3). In this case, we take $x_{0}=1$; then $r_{1}=0.289222 \cdots$.

\section{Acknowledgments}

This work is supported by the National Basic Research 973 Program of China (no. 2011JB105001), National Natural Science Foundation of China (Grant no. 11371320), the Foundation of Science and Technology Department (Grant no. 2013C31084) of Zhejiang Province, and the Foundation of the Education Department (nos. 20120040 and Y201329420) of Zhejiang Province of China and also by the Grant FEKT-S11-2-921 of Faculty of Electrical Engineering and Communication, Brno University of Technology, Czech Republic.

\section{References}

[1] J. M. Ortega and W. C. Rheinboldt, Iterative Solution of Nonlinear Equations in Several Variables, Academic Press, New York, NY, USA, 1970. 
[2] A. Cordero, J. L. Hueso, E. Martínez, and J. R. Torregrosa, "Increasing the convergence order of an iterative method for nonlinear systems," Applied Mathematics Letters, vol. 25, no. 12, pp. 2369-2374, 2012.

[3] A. Cordero, J. R. Torregrosa, and P. Vindel, "Study of the dynamics of third-order iterative methods on quadratic polynomials," International Journal of Computer Mathematics, vol. 89, no. 1314, pp. 1826-1836, 2012.

[4] L. V. Kantorovich, “On Newton's method," Akademiya Nauk SSSR. Trudy Matematicheskogo Instituta imeni V. A. Steklova, vol. 28, pp. 104-144, 1949 (Russian).

[5] Z. D. Huang, "A note on the Kantorovich theorem for Newton iteration," Journal of Computational and Applied Mathematics, vol. 47, no. 2, pp. 211-217, 1993.

[6] X. H. Wang and C. Li, "Convergence of Newton's method and uniqueness of the solution of equations in Banach spaces. II," Acta Mathematica Sinica, vol. 19, no. 2, pp. 405-412, 2003.

[7] I. K. Argyros, "On Newton's method for solving equations containing Fréchet-differentiable operators of order at least two," Applied Mathematics and Computation, vol. 215, no. 4, pp. 1553-1560, 2009.

[8] J. A. Ezquerro, J. M. Gutiérrez, M. A. Hernández, and M. A. Salanova, "The application of an inverse-free Jarratt-type approximation to nonlinear integral equations of Hammerstein-type," Computers \& Mathematics with Applications, vol. 36, no. 4, pp. 9-20, 1998.

[9] M. Chen, Y. Khan, Q. Wu, and A. Yildirim, "Newton-Kantorovich convergence theorem of a modified Newton's method under the gamma-condition in a Banach space," Journal of Optimization Theory and Applications, vol. 157, no. 3, pp. 651662, 2013.

[10] J. A. Ezquerro, D. González, and M. A. Hernández, "A modification of the classic conditions of Newton-Kantorovich for Newton's method," Mathematical and Computer Modelling, vol. 57, no. 3-4, pp. 584-594, 2013.

[11] J. A. Ezquerro, D. González, and M. A. Hernández, "A variant of the Newton-Kantorovich theorem for nonlinear integral equations of mixed Hammerstein type," Applied Mathematics and Computation, vol. 218, no. 18, pp. 9536-9546, 2012.

[12] M. Frontini and E. Sormani, "Modified Newton's method with third-order convergence and multiple roots," Journal of Computational and Applied Mathematics, vol. 156, no. 2, pp. 345-354, 2003.

[13] J. A. Ezquerro and M. A. Hernández, "On the R-order of convergence of Newton's method under mild differentiability conditions," Journal of Computational and Applied Mathematics, vol. 197, no. 1, pp. 53-61, 2006.

[14] S. Weerakoon and T. G. I. Fernando, "A variant of Newton's method with accelerated third-order convergence," Applied Mathematics Letters, vol. 13, no. 8, pp. 87-93, 2000.

[15] M. Frontini and E. Sormani, "Some variant of Newton's method with third-order convergence," Applied Mathematics and Computation, vol. 140, no. 2-3, pp. 419-426, 2003.

[16] M. Frontini and E. Sormani, "Third-order methods from quadrature formulae for solving systems of nonlinear equations," Applied Mathematics and Computation, vol. 149, no. 3, pp. 771-782, 2004.

[17] X. H. Wang and C. Li, "The local and global behaviors of methods for solving equations," Chinese Science Bulletin, vol. 46, no. 6 , pp. 444-451, 2001.
[18] Y. Zhao and Q. Wu, "Convergence analysis for a deformed Newton's method with third-order in Banach space under $\gamma$ condition," International Journal of Computer Mathematics, vol. 86, no. 3, pp. 441-450, 2009. 


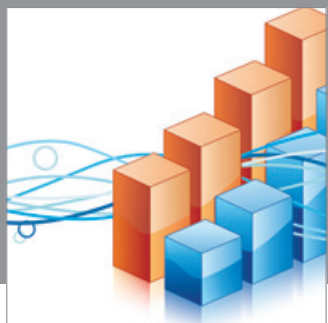

Advances in

Operations Research

mansans

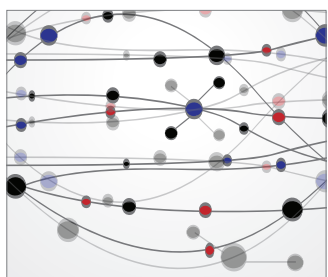

The Scientific World Journal
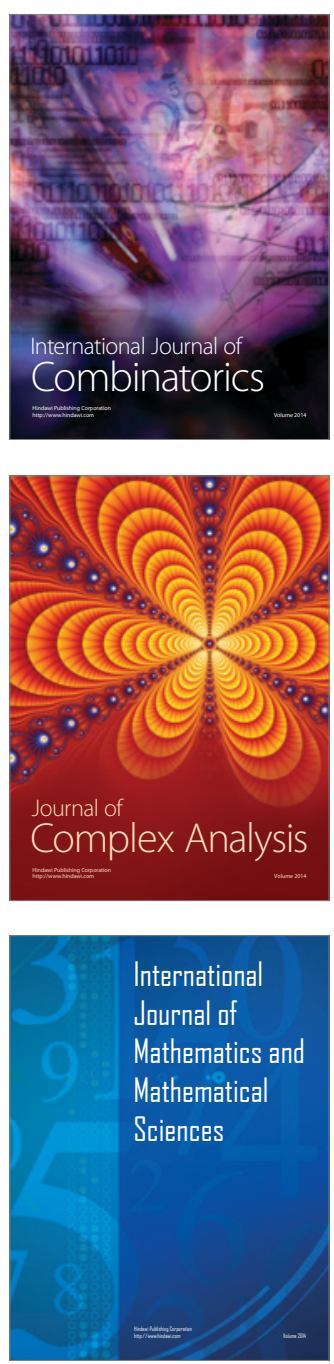
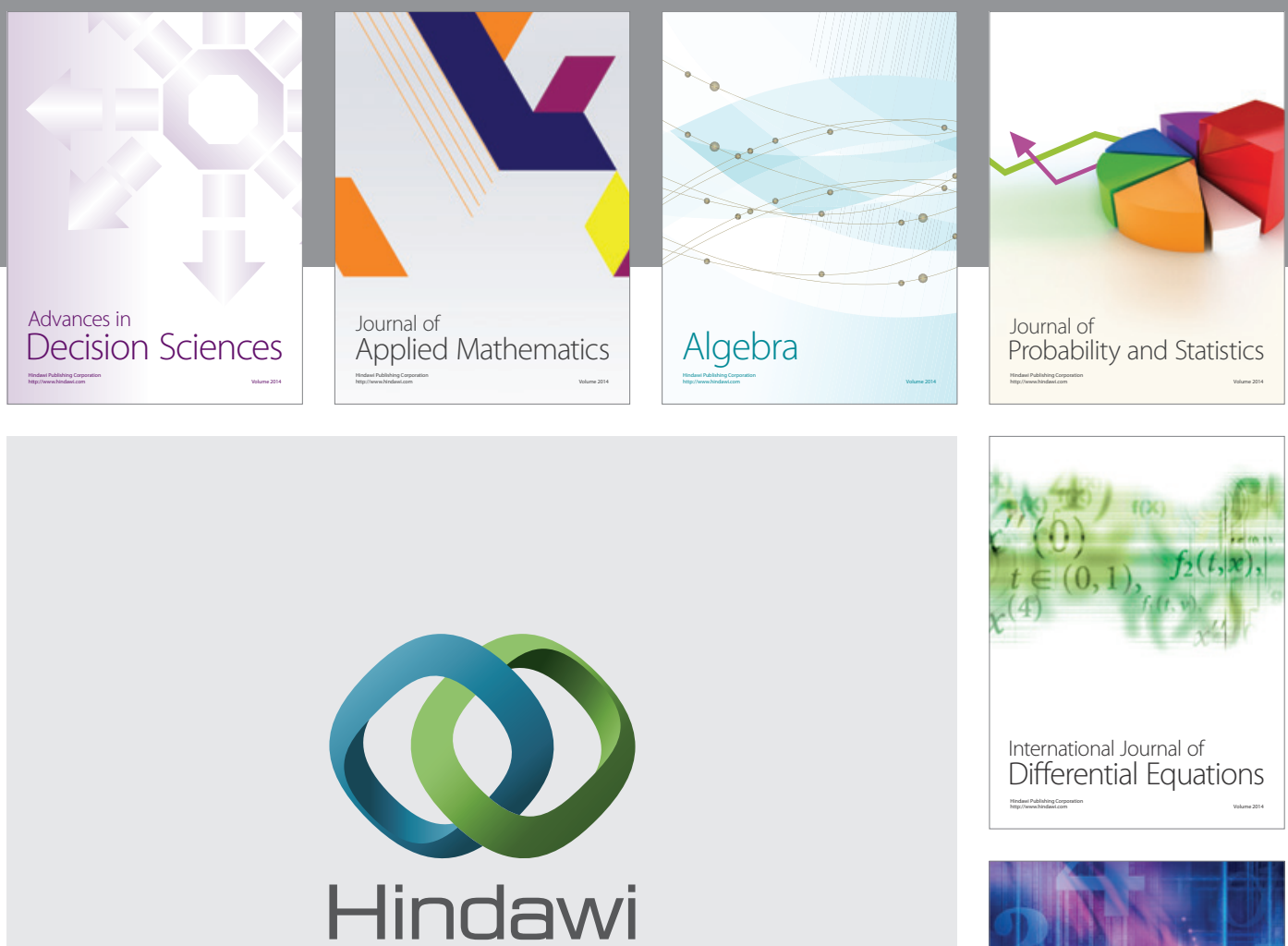

Submit your manuscripts at http://www.hindawi.com
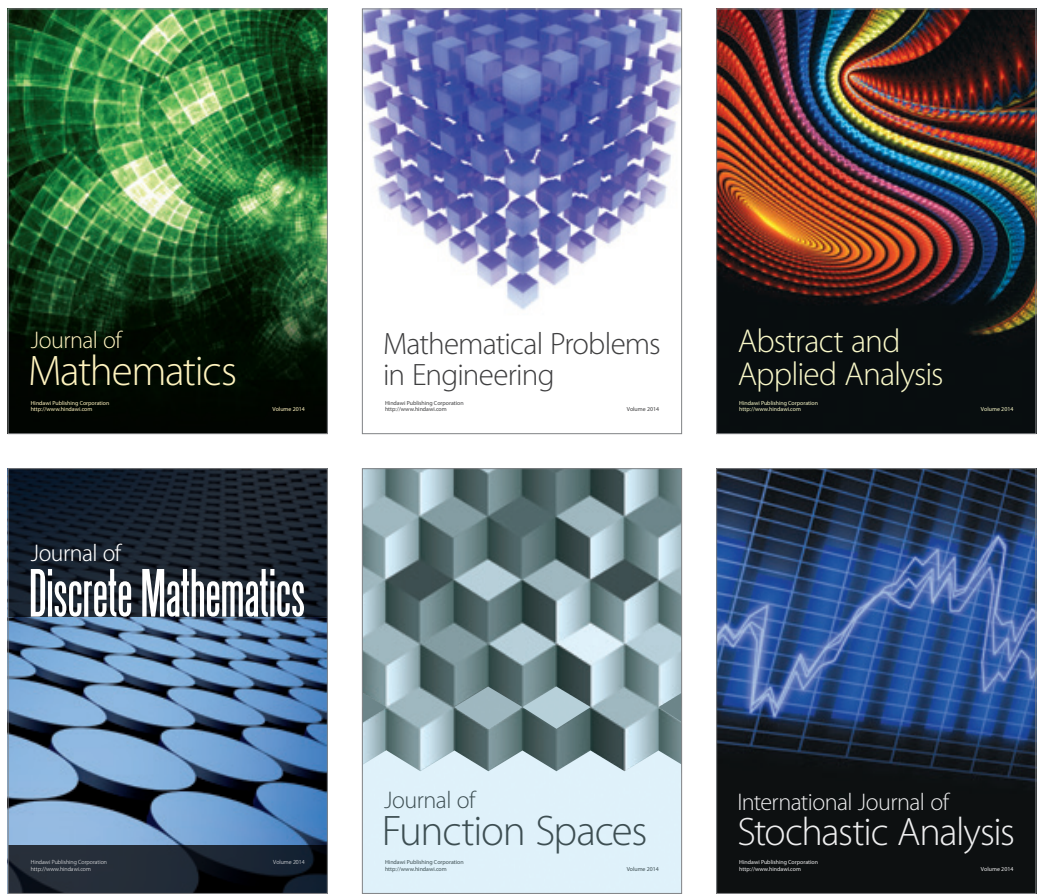

Journal of

Function Spaces

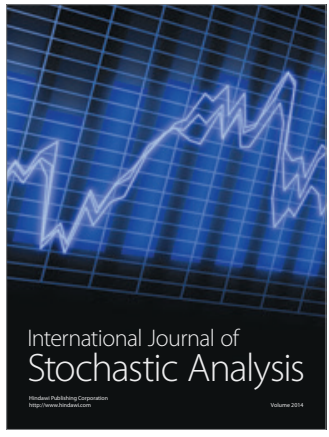

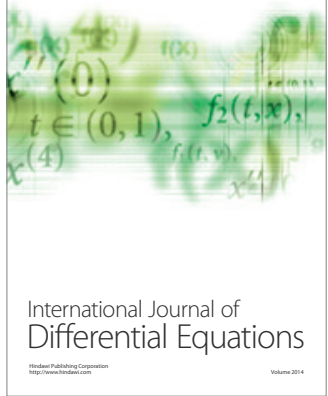
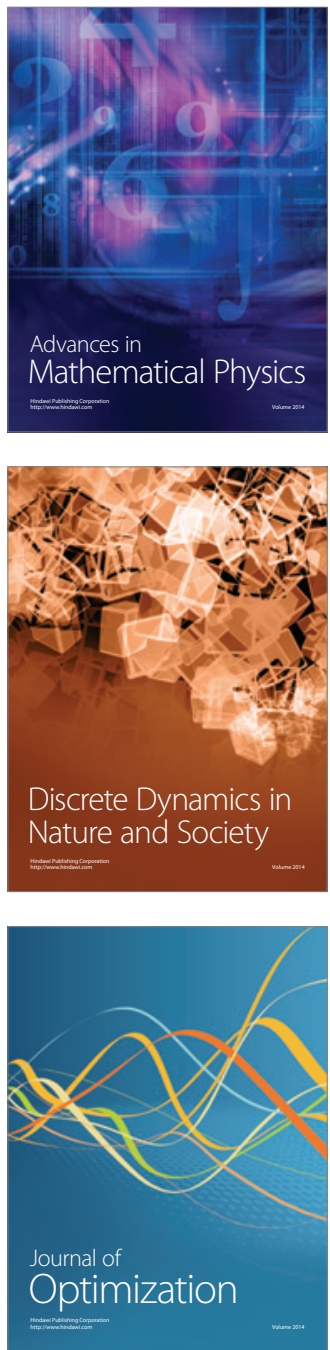